\title{
Natal kicks of stellar-mass black holes
}

\author{
Melvyn B. Davies ${ }^{* a}$, Serena Repetto ${ }^{a b}$ and Steinn Sigurdsson ${ }^{c}$ \\ ${ }^{a}$ Lund Observatory, Department of Astronomy and Theoretical Physics, Box 43, SE-22100, Lund, \\ Sweden. \\ ${ }^{b}$ Department of Astrophysics/IMAPP, Radbound University Nijmegen, P.O. Box 9010, 6500 GL \\ Nijmegen, The Netherlands. \\ ${ }^{c}$ Department of Astronomy \& Astrophysics, Pennsylvania State University, 525 Davey Lab, \\ University Park, PA 16802, USA. \\ E-mail: mbd@astro.lu.se, S.Repetto@astro.rul.nl, steinn@astro.psu.edu
}

\begin{abstract}
We investigate whether stellar-mass black holes have to receive natal kicks in order to explain the observed distribution of low-mass X-ray binaries containing black holes within our Galaxy. Such natal kicks will affect the production of compact BH-NS binaries which are possible GRB progenitors. We combine population synthesis with integrations of binary systems within the Galactic potential. We find that in a number of cases, natal kicks are in fact necessary. Further, we find that the distribution of natal kicks would seem to be similar to that of neutron stars. This result is somewhat surprising; in many pictures of stellar-mass black-hole formation, one might have expected black holes to receive kicks having the same momentum (rather than the same speed) as those given to neutron star.
\end{abstract}

Gamma-Ray Bursts 2012 Conference-GRB2012,

May 07-11, 2012

Munich, Germany

*Speaker. 


\section{Introduction}

Low-mass X-ray binaries containing black holes (BH-LMXBs) have been observed throughout the Galaxy. Their positions in cylindrical coordinates are shown in Figure 1. As seen in this figure, they are found at a variety of distances from the Galactic disc. These systems are thought to form from the evolution of massive stars within binaries and thus should form in the Galactic disc. Those reaching large heights from the disc must therefore have received some form of kick when the black hole was formed in a supernova explosion. This kick can have two components: one from the mass-loss associated with the supernova explosion and one due to any natal kick given to the black hole on its formation.

We have used population synthesis to produce a population of binary systems giving the binaries random kicks with a natal kick component drawn from various kick distributions. We then integrated the motion of the binaries within a model for the Galactic potential noting their positions at regular intervals so producing a synthetic distribution of X-ray binaries which can be compared to the observed population.

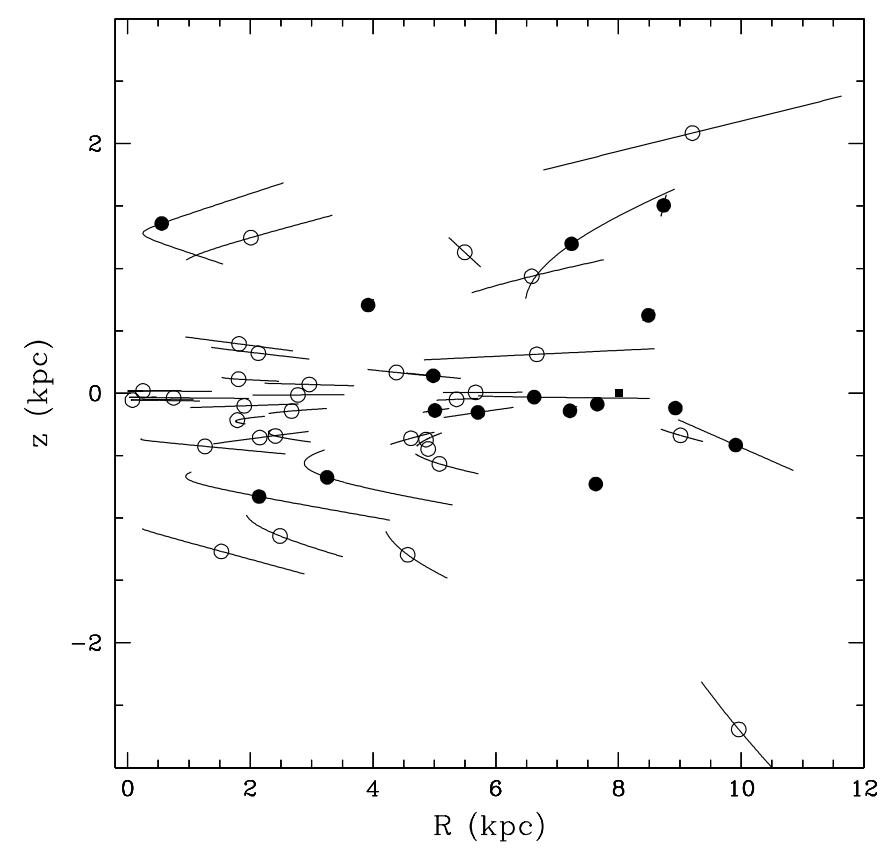

Figure 1: Galactic distribution, in cylindrical coordinates, of low-mass X-ray binaries containing neutron stars or black holes: NS-LMXBs (open circles) and BH-LMXBs (filled circles). Locations and distances have been taken from [1] and [2]. Lines denote position uncertainties.

\section{Results}

In Figure 2 we plot the positions in cylindrical coordinates of BH-LMXBs using various natal kick distributions. We find that natal kicks comparable to those inferred for neutron stars are necessary to produce a good match to the observed BH-LMXB distribution. 


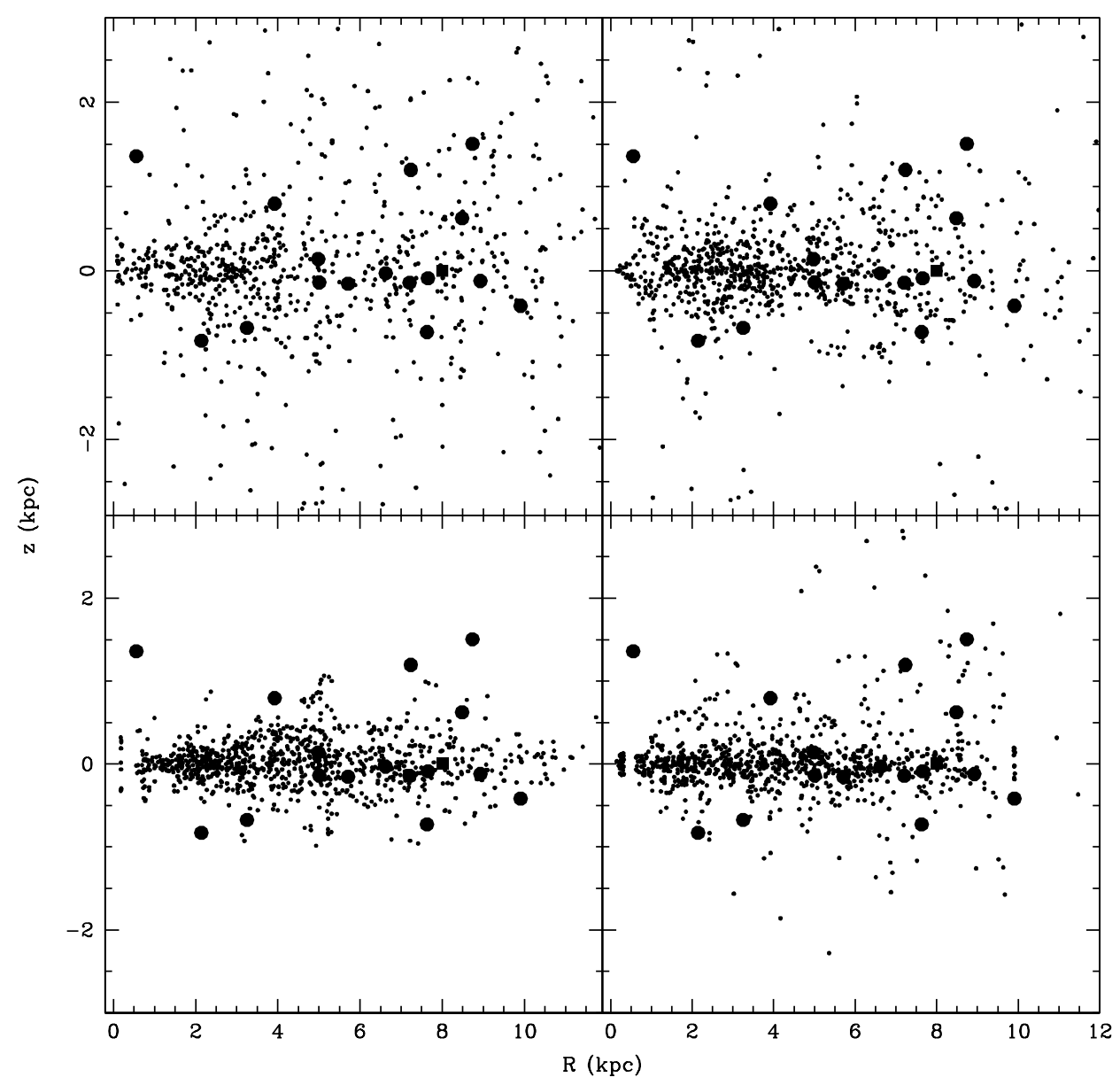

Figure 2: Binary population synthesis for a sample of BH-LMXBs. Natal kicks have been drawn from the Hansen \& Phinney kick distribution [3] (top left) or the Arzoumanian kick distribution [4] (top right), whereas the bottom figures correspond to using natal kicks reduced by the ratio of black hole to neutron star masses as would be expected if black holes are formed via fallback of material onto neutron stars. The synthetic population is shown as small dots whereas the observed systems are shown as large dots.

In Figure 3 we plot the cumulative distributions of BH-LMXBs as a function of distance from the Galactic plane. We see that the distribution of the synthetic population is a good match to the observed distribution for both the Hansen \& Phinney and Arzoumanian neutron-star kick distributions [3, 4]. A very poor fit is found for the case of zero natal kick. This is also true for kick distributions reduced by the ratio of black-hole to neutron-star masses.

\section{Conclusion}

We conclude that black holes seem likely to receive natal kicks comparable to those given to neutron stars. These results are presented in more detail in [5]. 


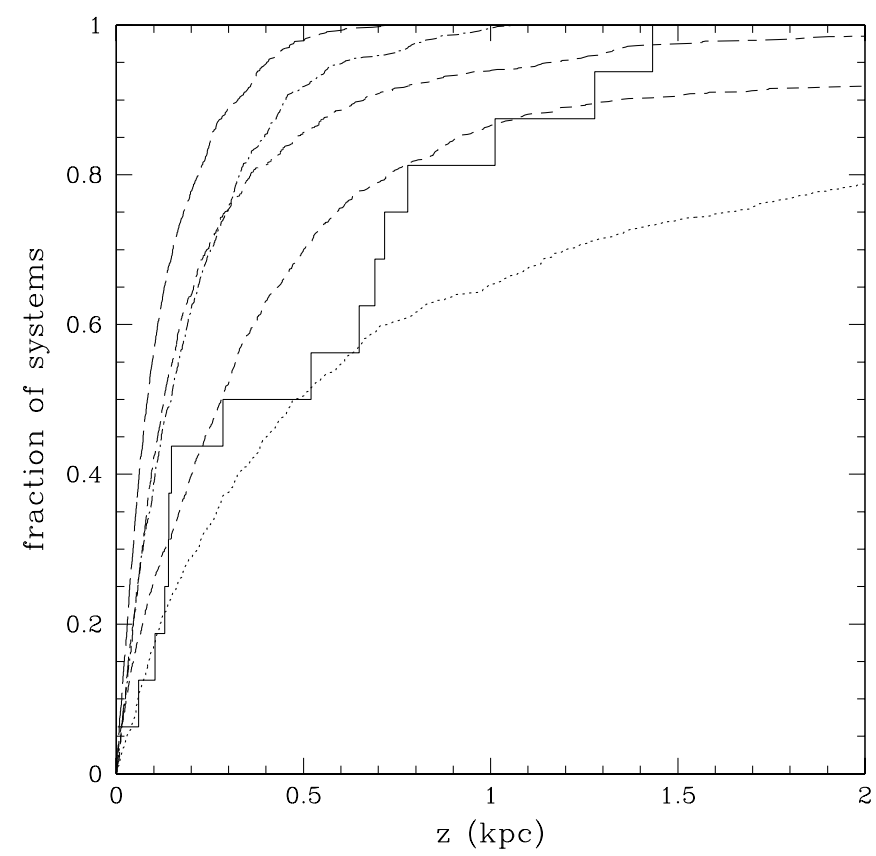

Figure 3: Cumulative distributions of BH-LMXBs as a function of distance from the Galactic plane, for the observed systems (solid line) and for our synthetic population using different natal kicks (dotted line is for a Hansen \& Phinney natal kick, dotted-dashed line is for a reduced Hansen \& Phinney natal kick, short-dashed is for an Arzoumanian natal kick, short-long-dashed is for a reduced Arzoumanian natal kick, whereas long-dashed is for zero natal kick).

\section{Acknowledgments}

This work was supported by the Swedish Research Council (grants 2008-4089 and 20113991). SR is thankful to the University of Pavia for the Erasmus grant and Andrea de Luca for helpful suggestions. MBD and SS gratefully acknowledge the hospitality of the Aspen Center for Physics.

\section{References}

[1] P.G. Jonker, G.Nelemans, The distances to Galactic low-mass X-ray binaries: consequences for black hole luminosities and kicks, MNRAS 354 (2004) 355

[2] F. Ozel, D. Psaltis, R. Narayan, J.E. McClintock, The Black Hole Mass Distribution in the Galaxy, ApJ 725 (2010) 1918

[3] B.M.S. Hansen, E.S.Phinney, The pulsar kick velocity distribution, MNRAS 291 (1997) 569

[4] Z. Arzoumanian, D.F. Chernoff, J.M.Cordes, The Velocity Distribution of Isolated Radio Pulsars, ApJ 568 (2002) 289

[5] M.B. Davies, S. Repetto, S. Sigurdsson, Investigating stellar-mass black hole kicks, MNRAS submitted [arXiv:1203.3077] 